\title{
Extensive myelitis associated with anti-NMDA receptor antibodies
}

\author{
Olivier Outteryck ${ }^{*}$, Guillaume Baille ${ }^{1}$, Jérôme Hodel ${ }^{2}$, Marianne Giroux ${ }^{1}$, Arnaud Lacour ${ }^{1}$, Jérôme Honnorat ${ }^{3,4,5}$, \\ Hélène Zéphir ${ }^{1}$ and Patrick Vermersch ${ }^{1}$
}

\begin{abstract}
Background: Encephalitis with anti-N-methyl-D-aspartate receptor antibodies (anti-NMDAR-Ab) is a rapid-onset encephalitis including psychosis, seizures, various movement disorders and autonomic system disturbances.

Case presentation: We report a very unusual case of extensive myelitis associated with anti-NMDAR-Ab. MRI also revealed a hyperintense T2 lesion, non-suggestive of MS, which progressively extended, associated with periventricular gadolinium enhancement visualized on brain MRI. Ophthalmological evaluation showed subclinical right optic neuritis. The absence of anti-AQP4 antibody argued against neuromyelitis optica spectrum disorder. A slight psychomotor slowing prompted us to search for various causes of autoimmune encephalitis. Anti-NMDAR-Ab was found in cerebrospinal fluid.
\end{abstract}

Conclusion: In patients with extensive myelitis who are seronegative for anti-AQP4 antibodies, and after other classical causes have been excluded, the hypothesis of atypical anti-NMDAR-Ab encephalitis should also be considered.

Keywords: Myelitis, Anti-NMDAR antibodies, Encephalitis, Neuromyelitis optica

\section{Background}

Encephalitis with anti-N-methyl-D-aspartate receptor antibodies (anti-NMDAR-Ab) is a rapid-onset encephalitis including psychosis, seizures, various movement disorders and autonomic system disturbances. The physiopathology is based on immune-mediated neuronal dysfunction [1,2]. We report the case of a 65 -year-old woman presenting an extensive myelitis associated with anti-NMDAR-Ab.

\section{Case presentation}

A 65-year-old woman was admitted because of progressive paraparesis (June 2012). Six months before admission, she had presented with fever, chills, abdominal pain and unusual headache for one week with spontaneous recovery. Abdomen and brain MRI were normal at that time. Two months before admission, she described a progressive onset of walking difficulties. At the same time, constipation and dysuria were noted. At her admission, neurological examination noted walking difficulties related to moderate paraparesis, moderate superficial and deep sensory

\footnotetext{
* Correspondence: olivier.outteryck@chru-lille.fr

'Department of Neurology, EA2686, Université Lille Nord de France, Lille, France

Full list of author information is available at the end of the article
}

dysfunction of the lower limbs and urinary retention. A very slight dysfunction of mental processing without speech disturbance was noted and confirmed by her family. No seizures, dyskinesia, movement disorders, psychiatric symptoms or autonomic dysfunction were observed.

Spinal cord MRI showed longitudinally extensive myelitis from C5 to T10 with gadolinium (Gd) enhancement (Figure 1A,B). Brain MRI follow-up showed T2 hyperintensities within the insular regions, medial temporal lobes and thalamus (Figure 1C), associated with gadolinium enhancement of the meninges and ventricles (Figure 1D). Analysis of the cerebrospinal fluid (CSF) showed moderate lymphocytic pleocytosis $\left(53\right.$ cells $\left./ \mathrm{mm}^{3}\right)$, mild increased protein concentration $(0.91 \mathrm{~g} / \mathrm{L})$ and oligoclonal bands. PCR assays for herpesviridae in CSF were all negative. Ophthalmological examination showed delayed P100 latencies on the right side on visual evoked potentials and asymmetric global retinal nerve fibre layer (RNFL) thicknesses on optical coherence tomography (OCT) suggestive of asymptomatic right optic neuritis $(\mathrm{ON})$. OCT analyses were both perfectly centred on the optic nerve head (Figure 1E). Tests for anti-aquaporin 4 $(\mathrm{AQP} 4)$ antibodies $(\mathrm{Ab})$ in serum were negative. EEG 


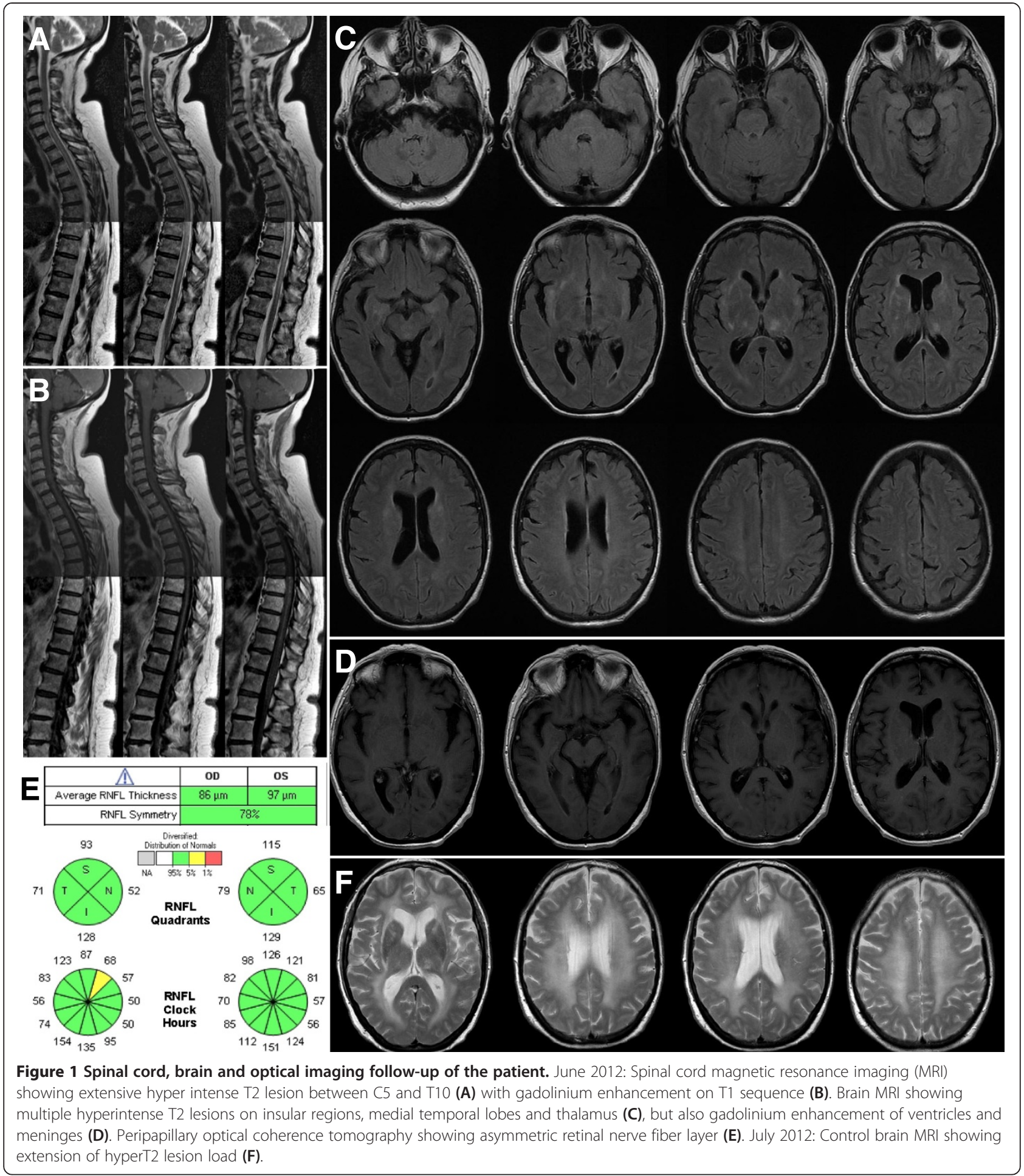

showed slow and discontinuous activity in the left fronto-temporal regions. Extensive tests for auto- $\mathrm{Ab}$ (anti-onconeuronal, anti-DNA, anti-phospholipid, antivoltage-gated potassium channel, anti-glutamate acid decarboxylase) were negative except for anti-NMDAR$\mathrm{Ab}$ (IgG isotype) in the CSF and serum. No cancer was found (gynaecological examination, pelvic ultrasonography and MRI, mammography, total-body computed tomography scan and positron emission tomography scan were all normal).

Intravenous corticosteroids $(1 \mathrm{~g}$ per day during 3 days plus 5 days) were given but the paraparesis worsened 
(bedridden patient). Brain and spinal cord MRIs follow-up (July 2012) showed an extension of T2 lesions without Gd enhancement (Figure 1F) and a stable extensive T2 lesion without Gd enhancement, respectively. As soon as antiNMDAR-Ab were detected, plasma exchanges (PLEX) were initiated and followed by intravenous rituximab (375 mg/m ${ }^{2}$ every week for 4 weeks) plus tapering oral corticosteroids $(1 \mathrm{mg} / \mathrm{kg})$. Brain and spinal cord MRIs follow-up (August 2012) showed the disappearance of the extensive spinal cord T2 lesion and stable cerebral T2 lesions. Biological follow-up of anti-NMDAR-Ab rates in CSF and serum was performed (Table 1). CSF analysis was weakly positive just after corticosteroids/ PLEX (M1) and was negative 2 months after rituximab (M3). Serum analysis was less correlated to clinical status. Four months after the inaugural myelitis, there was a significant improvement of the paraparesis (the patient was able to walk for 5 metres with bilateral support) and a total regression of the slight psychomotor retardation was observed. Unfortunately, the patient presented rapidly evolving pneumocystis pneumonia at this time and she died of respiratory failure. Autopsy was not performed.

\section{Discussion}

Our patient presented extensive myelitis associated with anti-NMDAR-Ab leading us to discuss a possible atypical form of anti-NMDAR-Ab encephalitis. Spinal cord symptoms were prominent throughout the clinical stage and were consistent with the extensive myelitis detected on spinal cord MRI. Slight psychomotor retardation is not sufficient to affirm that our patient presented antiNMDAR-Ab encephalitis but early treatment might have stopped its development. Brain MRI showed hyperintense T2 lesions but also ventriculitis aspect. In view of the combination of extensive myelitis, brain T2 lesions non-suggestive of MS, ventriculitis aspect on MRI and subclinical ON, our first hypothesis was myelitis as a first event of neuromyelitis optica (NMO) or NMO spectrum disorder (NMOSD). Although cognitive disturbances are described in NMO [3], we also looked for other conditions involving auto-immune encephalitis and found anti-NMDAR-Ab in the CSF before immunosuppressive drugs had been applied.

Prodromal symptoms such as headache and fever are common [1] but we noted 3 atypical events for the hypothesis of anti-NMDAR-Ab encephalitis. First, myelitis is a very rare manifestation during anti-NMDAR-Ab encephalitis but has already been described [4-6]. In these 3 previous cases, all patients presented severe psychiatric or behavioural symptoms before the spinal cord syndrome and the encephalopathic symptoms remained prominent during all stages of the disease. In our case, the spinal cord syndrome remained prominent from the beginning and extension of the hyperintense T2 lesion was greater. Secondly, we highlighted in our case ventriculitis associated with meningeal Gd enhancement. Meningeal contrast enhancement in anti-NMDAR-Ab encephalitis is rare and $[7,8]$, to the best of our knowledge, the ventriculitis aspect has never previously been described. NMDA receptors are membrane receptors widely expressed in the central nervous system on various cells such as neurons, oligodendrocytes but also astrocytes [9]. The ependymal regions contain ependymal cells and astrocytes rich in AQP4, and can also be involved in $\mathrm{NMO}$, as described in 2 previously reported cases with thin ependymal and periventricular Gd enhancement $[10,11]$. Thirdly, our patient presented right subclinical ON. Since the prodromal symptoms dated back 6 months, it seems likely that the demyelinating pathology had already started 6 months before the spinal cord syndrome. RNFL atrophy most often $(>94 \%)$ appears within the 6 months after ON [12]. ON has only once been reported as a neurological manifestation of anti-NMDAR-Ab encephalitis [13].

Even though some correlations have been found between clinical status and anti-NMDAR-Ab titres in CSF, there is no reported correlation with anti-NMDAR-Ab titres in serum, and we therefore do not recommend a follow-up of anti-NMDAR-Ab titres in serum $[1,14]$.

Anti-NMDAR-Ab encephalitis has been described following massive brain damage induced by herpes simplex

Table 1 Anti-NMDA-R follow-up of the titration in CSF and in serum (qualitative and quantitative)

\begin{tabular}{|c|c|c|c|c|c|c|c|c|c|c|c|c|}
\hline Date & $\begin{array}{l}\text { Jun } \\
23 r d\end{array}$ & $\begin{array}{l}\text { Jul } \\
\text { 2nd }\end{array}$ & $\begin{array}{c}\text { Jul } \\
10 \text { th }\end{array}$ & $\begin{array}{c}\text { Jul } \\
11 \text { th }\end{array}$ & $\begin{array}{c}\text { Jul } \\
14 \text { th }\end{array}$ & $\begin{array}{c}\text { Jul } \\
16 \text { th }\end{array}$ & $\begin{array}{c}\text { Jul } \\
17 \text { th }\end{array}$ & $\begin{array}{c}\text { Jul } \\
23 r d\end{array}$ & $\begin{array}{c}\text { Jul } \\
30 \text { th }\end{array}$ & $\begin{array}{l}\text { Aug } \\
6 \text { th }\end{array}$ & $\begin{array}{l}\text { Aug } \\
13 \text { th }\end{array}$ & $\begin{array}{l}\text { Oct } \\
17 \text { th }\end{array}$ \\
\hline CSF & $+(1 / 10)$ & $+($ low $)$ & NA & NA & NA & NA & - & NA & NA & NA & NA & - \\
\hline Anti-NMDA-R in serum & NA & NA & $+(1 / 10)$ & $+(1 / 10)$ & - & $+($ low $)$ & - & - & $+($ low $)$ & NA & $+(1 / 10)$ & - \\
\hline $\begin{array}{l}\text { Methylprednisolone } \\
\text { (1 g/day IV) }\end{array}$ & 3 days & & 5 days & & & & & & & & & \\
\hline PLEX & & & Yes & Yes & Yes & Yes & Yes & & & & & \\
\hline $\begin{array}{l}\text { Rituximab ( } 375 \text { mg/m2/week } \\
\text { during } 4 \text { weeks) }\end{array}$ & & & & & & & & Yes & Yes & Yes & Yes & \\
\hline
\end{tabular}

Jun, June; Jul, July; Aug, August; Oct, October; CSF, cerebrospinal fluid; NA, not available; PLEX, plasma exchange; 5 PLEX were performed followed by infusions of rituximab (375 mg/m2/week during 4 weeks). 
encephalitis (HSE) [15,16]. Anti-NMDAR-Ab has also been found in sera of systemic lupus erythematosus and might be predictive of neuropsychiatric manifestations [17]. In the present case report, we cannot affirm that our patient presented anti-NMDAR-Ab encephalitis because of encephalopathic symptoms lacking and also cannot exclude that a massive brain and spinal damage of unknown etiology has led to secondary immunological response with anti-NMDA-R Ab production.

We can also discuss the hypothesis that our patient may have presented 2 auto-immune diseases concurrently: an NMOSD and an anti-NMDAR-Ab encephalitis. These are both Ab-mediated diseases and a comorbid association of auto-immune diseases is possible. Recently a case of NMO (seropositive anti-AQP4 Ab) following anti-NMDAR-Ab encephalitis has been described [18]. As our patient was seronegative for anti-AQP4 Ab and presented only subclinical ON, we cannot conclusively confirm or rule out this hypothesis.

\section{Conclusion}

In patients with extensive myelitis who are seronegative for anti-AQP4 Ab, and after other classical causes have been excluded, the hypothesis of atypical anti-NMDARAb encephalitis should also be considered.

\section{Consent}

Written informed consent was obtained from the husband of the patient for publication of this Case report and the accompanying images. A copy of the written consent is available for review by the editor of this journal.

\section{Competing interests}

The authors declare that they have no competing interests.

\begin{abstract}
Authors' contributions
○० drafted the manuscript, participated to acquisition, analysis and interpretation of the data. GB participated to drafting and acquisition of data. JHod participated to acquisition of data and revised the manuscript for important intellectual concept. MG participated to acquisition of data and revised the manuscript for important intellectual concept. AL participated to acquisition of data and revised the manuscript for important intellectual concept. JHon revised the manuscript for important intellectual concept. HZ participated to analysis and interpretation of data and revised the manuscript for important intellectual concept. PV participated to acquisition of data and revised the manuscript for important intellectual concept. All authors read and approved the manuscript.
\end{abstract}

\footnotetext{
Author details

'Department of Neurology, EA2686, Université Lille Nord de France, Lille, France. ${ }^{2}$ Department of Neuroradiology, Université Lille Nord de France, Lille, France. ${ }^{3}$ French Reference Centre for Paraneoplastic Neurological Syndrome, Hospices Civils de Lyon, Hôpital Neurologique, F-69677, Bron, France. ${ }^{4}$ Lyon Neuroscience Research Center INSERM U1028/CNRS UMR 5292, F-69372, Lyon, France. ${ }^{5}$ Université de Lyon, Université Claude Bernard Lyon 1, F-69372, Lyon, France.
}

\section{References}

1. Dalmau J, Lancaster E, Martinez-Hernandez E, et al: Clinical experience and laboratory investigations in patients with anti-NMDAR encephalitis. Lancet Neurol 2011, 10:63-74.

2. Misakova L, De Rossi $P$, Bouchet $D$, et al: Disrupted surface cross-talk between NMDA and Ephrin-B2 receptors in anti-NMDA encephalitis. Brain 2012, 135:1606-1621.

3. Blanc F, Zéphir $H$, Lebrun $C$, et al: Cognitive functions in neuromyelitis optica. Arch Neurol 2008, 65:84-88.

4. Taylor RB, Mason W, Kong K, et al: Reversible paraneoplastic encephalomyelitis associated with a benign ovarian teratoma. Can J neurol Sci 1999, 26:317-320.

5. Kruer MC, Koch TK, Bourdette DN, et al: NMDA receptor encephalitis mimicking seronegative neuromyelitis optica. Neurology 2010, 74:1473-1475.

6. Lekoubou A, Viaccoz A, Didelot A, et al: Anti-N-methyl-D-aspartate receptor encephalitis with acute disseminated encephalomyelitis-like MRI features. Eur J Neurol 2012, 19:e16-e17.

7. Dalmau J, Tüzün E, Wu HY, et al: Paraneoplastic anti-N-methyl-D-aspartate receptor encephalitis associated with ovarian teratoma. Ann Neurol 2007, 61:25-36.

8. Dalmau J, Gleichman AJ, Hughes EG, et al: Anti-NMDA-receptor encephalitis: case series and analysis of the effect of antibodies. Lancet Neurol 2008, 7:1091-1098.

9. Paoletti P, Bellone C, Zhou Q: NMDA receptor subunit diversity: impact on receptor properties synaptic plasticity and disease. Nat Rev Neurosci 2013, 14:383-400.

10. Pittock SJ, Weinshenker BG, Lucchinetti CF, et al: Neuromyelitis optica brain lesions localized at sites of high aquaporin 4 expression. Arch Neurol 2006, 63:964-968.

11. Banker $P$, Sonni $S$, Kister I, et al: Pencil-thin ependymal enhancement in neuromyelitis optica spectrum disorders. Mult Scler 2012, 18:1050-1053.

12. Costello F, Coupland S, Hodge W, et al: Quantifying axonal loss after optic neuritis with optical coherence tomography. Ann Neurol 2006, 59:963-969.

13. Ishikawa N, Tajima G, Hyodo S, et al: Detection of autoantibodies against NMDA-type glutamate receptor in a patient with recurrent optic neuritis and transient cerebral lesions. Neuropediatrics 2007, 38:257-260.

14. Frechette ES, Zhou L, Galetta SL, et al: Prolonged follow-up and CSF antibody titers in a patient with anti-NMDA receptor encephalitis. Neurology 2011, 76:S64-S66.

15. Pruss H, Finke C, Höltje M, Hofmann J, Klingbeil C, Probst C, Borowski K, Ahnert-Hilger G, Harms L, Scwab JM, Ploner CJ, Koromowski L, Stoecker W, Dalmau J, Wandiger KP: N-methyl-D-aspartate receptor antibodies in herpes simplex encephalitis. Ann Neurol 2012, 72:902-911.

16. Armangue T, Leypoldt F, Málaga I, Raspall-Chaure M, Marti I, Nichter C, et al: Herpes Simplex Virus Encephalitis is a Trigger of Brain Autoimmunity. Ann Neurol 2013. doi:10.1002/ana.24083. epub ahead of print.

17. Gono T, Kawaguchi Y, Kaneko H, Nishimura K, Hanaoka M, Kataoka S, et al: Anti-NR2A antibody as a predictor for neuropsychiatric systemic lupus erythematosus. Rheumatology 2011, 50:1578-1585.

18. Zoccarato M, Saddi MV, Serra G, Pelizza MF, Rosellini I, Peddone L, Ticca A, Giometto B, Zuliani L: Aquaporin-4 antibody neuromyelitis optica following anti-NMDA receptor encephalitis. J Neurol 2013, 260:3185-3187.

\section{doi:10.1186/1471-2377-13-211}

Cite this article as: Outteryck et al.: Extensive myelitis associated with anti-NMDA receptor antibodies. BMC Neurology 2013 13:211. 